\title{
The combination of epidermal growth factor and glycogen synthase kinase 3 inhibitor support long-term self-renewal of Sca-1 positive hepatic progenitor cells from normal adult mice
}

\author{
Cai-Xia Jin ${ }^{1,2}$, Lisa Samuelson ${ }^{1}$, Cai-Bin Cui ${ }^{1}$, Yang-Zhong Sun ${ }^{1}$, David A. Gerber ${ }^{1,3^{*}}$ \\ ${ }^{1}$ Department of Surgery, University of North Carolina at Chapel Hill, Chapel Hill, USA; \\ *Corresponding Author: david gerber@med.unc.edu \\ ${ }^{2}$ Department of Regenerative Medicine and Huadong Stem Cell Bank, Tongji University School of Medicine, Shanghai, China \\ ${ }^{3}$ Lineberger Cancer Center, University of North Carolina at Chapel Hill, Chapel Hill, USA
}

Received 13 March 2013; revised 13 April 2013; accepted 13 May 2013

Copyright (C) 2013 Cai-Xia Jin et al. This is an open access article distributed under the Creative Commons Attribution License, which permits unrestricted use, distribution, and reproduction in any medium, provided the original work is properly cited.

\section{ABSTRACT}

Isolation and long-term maintenance of hepatic progenitor cells (HPCs) from healthy, non-injured adult livers remains challenging due to the lack of specific surface markers for selection and a limited understanding of the mechanisms for maintaining self-renewal. Previously, we identified a Sca-1 positive, bipotent HPC population in the peri-portal region of adult liver, and found MAPK/ERK and Wnt/ $\beta$-Catenin pathways to be synergistically involved in their proliferation. In this study, we report the long-term culture of Sca-1 positive HPCs with epidermal growth factor (EGF) and CHIR99021, a small molecule inhibitor of glycogen synthase kinase 3 (GSK-3). Sca-1+ HPCs remain non-tumorigenic when passaged 35 times in vitro over 1 year. Flow cytometric analysis indicates that HPCs are positive for Sca-1 and putative liver progenitor cell markers, including CD13, CD24 and Prominin-1, but negative for hematopoietic/endothelial cell markers CD31, CD34, CD45, CD90 and CD117. Immunocyto-chemistry and RT-PCR indicate Sca-1+ HPCs express albumin (ALB), $\alpha$ fetoprotein (AFP), cytokeratin19 (CK19), Sox9 and a panel of special hepatic progenitor transcriptional factors. Moreover, Sca-1+ HPCs are able to differentiate into hepatocyte-like and cholangiocyte-like cells under appropriate culture conditions in vitro and can take part in liver repopulation in an acetaminophen (APAP) induced liver injury mouse model. This study pro- vides a paradigm to capture and maintain HPCs from naïve liver tissue and offers a valuable cell model for investigating the molecular mechanisms underlying the cell lineage relationship in normal liver.

Keywords: Liver Progenitor Cell; Stem Cell Antigen 1; Liver Disease; Hematopoietic Stem Cell

\section{INTRODUCTION}

The liver's post-injury regenerative capability is well described. Previous studies suggest that self-duplication of mature hepatocytes is enough to maintain physiological tissue homeostasis and regenerate the liver after injury, such as $2 / 3$ hepatectomy. However, when the proliferative ability of mature hepatocytes is inhibited, "oval cells" are induced and participate in liver regeneration by differentiating into both hepatocytes and cholangiocytes [1-6]. Oval cells are not present in normal liver and are thought to be the descendant of putative endogenous hepatic progenitor cells (HPCs). However, despite extensive investigation of oval cells and ductal reaction after liver injury [5-9], there is limited knowledge about the naïve HPCs and their role in physiological and pathological liver regeneration.

Unique and discriminative surface markers that can be utilized for HPC isolation and identification have yet to be definitively reconciled. Several stem cell associated markers have generated interest in non-hematopoietic stem cell research. The murine stem cell antigen, Sca-1, is highly expressed on hematopoietic stem cells (HSC). Interestingly, Petersen et al. demonstrated Sca-1 expres- 
sion on murine oval cells in the 3,5-diethoxycarbonyl-1, 4-dihydrocollidine (DDC) liver injury model [10]. Previously, we identified a Sca-1 positive, bipotent HPC population in the peri-portal region of mouse liver [4]. By magnetic activated cell sorting (MACS), these Sca-1 positive HPC cells can be purified and demonstrate bipotent differentiation [4]. Recently, we found that MAPK/ ERK and $\mathrm{Wnt} / \beta$-Catenin pathways are synergistically involved in proliferation of Sca-1 positive HPCs [11]. In the current study we report the long-term maintenance and culture of Sca-1 positive HPCs with epidermal growth factor (EGF) and CHIR99021, a small molecule inhibitor of glycogen synthase kinase 3 (GSK-3). CHIR99021 works via activation of the canonical Wnt pathway by inhibiting GSK-3 $\beta$-dependent phosphorylation of $\beta$-Catenin [12]. Sca-1+ HPCs can be maintained for over 30 passages in vitro $>1$ year under such conditions while remaining non-tumorigenic. Even after long-term in vitro culture, HPCs remain bipotent and can generate functional hepatocytes in vivo.

Our study provides a new thread to molecular identification of HPCs from naïve mature liver tissue using Sca-1 as a marker. These Sca-1+ HPCs could be a useful cell model to investigate physiological liver regeneration and the self-renewal mechanisms of naïve HPCs.

\section{MATERIALS AND METHODS}

\subsection{Mice}

C57BL/6 mice and Rag2-/- $\gamma-/-$ mice were purchased from Jackson Laboratory (Bar Harbor, ME) and used for all of the experiments. All animal care and use protocols were approved by the Institutional Animal Care and Use Committee at the University of North Carolina at Chapel Hill in accordance with principles and procedures outlined in the National Institutes of Health Guide for the Care and Use of Laboratory Animals.

\subsection{Sca- $1^{+}$HPCs Isolation and Culture}

Sca-1+ HPCs were isolated from 6 - 8 week old C57BL/6 mice using a modification of the 2-stage liver perfusion technique as described [4,11,13]. Enrichment of Sca-1+ HPCs was performed using a Sca-1 antibody conjugated to mini-magnetic beads (Miltenyi Biotec, Inc.; Auburn, CA) according to the manufacturer's instructions. The cells were regularly maintained in Dulbecco's modified Eagle medium (DMEM) (Gibco; Carlsbad, California) with $10 \%$ fetal bovine serum (FBS) (PAA Laboratories, Inc.; Dartmouth, MA), $20 \mathrm{mM}$ hepes (Sigma Chemical Co.; St. Louis, MO), $10 \mathrm{mM}$ nicotinamide (Sigma), $1 \mathrm{mM}$ ascorbic acid 2-phosphate (Sigma), $30 \mathrm{mg} / \mathrm{L}$ L-proline (Sigma), $10 \mathrm{ng} / \mathrm{mL}$ EGF (Sigma) and $2 \mu \mathrm{M}$ CHIR99021 (Stemgent; San Diego, CA). The 9th passage Sca-1+ HPCs were serially diluted and plated onto 96-well plates for subcloning. Two weeks later, 10 isolated clones were selected, dissociated and expanded. Among these ten clonal lines, lines \#2, \#4, \#8 and \#10 demonstrated homogeneous epithelial cells, while the other lines expressed a mixed cellular morphology. All ten cultures have been stably cultured in vitro for more than 30 passages without a decrease in proliferation. Two clones were selected for extensive analysis in this study. They were labeled Clone 9-1, clone 9-8 and they were compared with the heterogeneous initial population of mixed HPCs.

\subsection{Cell Surface Markers and Cell Proliferation Analysis by Flow Cytometry}

Cells were dissociated into a single cell suspension with $0.25 \%$ trypsin (Sigma) and stained with primary antibodies labeled with relevant fluoroprobes for $20 \mathrm{~min}$ at room temperature at $1 \mu \mathrm{g}$ antibody per $1,000,000$ cells in DPBS. After three washes with DPBS, cells were resuspended in cold DPBS buffer containing $1 \%$ FBS at 1 $\times 10^{6}$ cells $/ \mathrm{ml}$. Unstained cells and cells stained with isotype control antibody were used as blank and negative controls. The directly conjugated primary antibodies included phycoerythrin (PE)-conjugated Sca-1, CD31, CD45 and CD117 (BD Pharmingen ${ }^{\mathrm{TM}}$, San Diego, CA) and Prominin-1 (Miltenyi Biotec, Inc.), PE-Cy7-conjugated EpCAM (eBiosciences; San Diego, CA), fluorescein isothiocyanate (FITC)-conjugated CD13, CD24, CD34, CD90 (Pharmingen). The fluorescence-labeled cells were analyzed with Cyan ADP (Dako) or LSR II flow cytometer (Beckman Coulter, Inc. Brea, CA). Debris and doublets were excluded by forward scatter and side scatter manipulation. Gating was implemented based on isotype control staining profiles. All data were analyzed with Summit 4.3 Software (Dako).

\subsection{RNA Isolation and RT-PCR}

Total RNA was extracted from HPCs, 9-1 HPCs and 9-8 HPCs by RNeasy Plus Mini Kit (Qiagen, Inc., Valencia, CA). One microgram of total RNA were reversetranscribed using the RETRO script Reverse Transcription Kit (Ambion; Austin, TX). PCR conditions were $95^{\circ} \mathrm{C}$ for $5 \mathrm{~min}, 94^{\circ} \mathrm{C}$ for $30 \mathrm{sec}$, annealing temperature for $40 \mathrm{sec}$, and $72^{\circ} \mathrm{C}$ for $60 \mathrm{sec}, 35-40$ cycles, and then $72^{\circ} \mathrm{C}$ for $10 \mathrm{~min}$.

\subsection{Western Blot Analysis}

Cells were harvested and homogenized in RIPA buffer (Sigma Aldrich; St Louis, MO) containing $1 \mathrm{ml}$ protease inhibitor cocktail and $1 \mathrm{ml}$ phosphatase inhibitor per 100 $\mathrm{ml}$. Lysates were centrifuged at 14,000 g for $2 \mathrm{~min}$. Protein concentrations of supernatants were determined by 
using a standard Bradford assay on a BioTek microplate reader. Equivalent amounts of $50 \mu \mathrm{g}$ protein were heating at $95^{\circ} \mathrm{C}$ for $10 \mathrm{~min}$ and ran on SDS-PAGE, transferred to nitrocellulose membrane, incubated with 5\% nonfat milk blocking buffer for $2 \mathrm{~h}$ at room temperature and probed with primary antibodies overnight at $4^{\circ} \mathrm{C}$. After incubation with peroxidase-conjugated secondary antibodies at room temperature for $1 \mathrm{~h}$, activity was detected by ECL $^{\text {TM }}$ Western Blotting Detection Reagents (Amersham). The primary antibodies of $\beta$-Actin and AFP were purchased from Sigma, ALB and CK19 were purchased from Dako (Carpinteria, CA), E-Cadherin was purchased from Cell Signaling Technology (Beverly, MA). Horseradish Peroxidase conjugated secondary antibodies were purchased from Dako.

\subsection{Immunofluorescence Assay}

Cells were fixed in 4\% paraformaldehyde for $30 \mathrm{~min}$ at room temperature (RT) and washed in PBS with $0.05 \%$ Tween-20 (T-PBS) three times, followed by blocking with $5 \%$ normal goat serum for $30 \mathrm{~min}$, then incubated with primary antibodies at $4^{\circ} \mathrm{C}$ overnight, washed three times in T-PBS, then incubated with second antibody for $30 \mathrm{~min}$. The following primary antibodies were used: Rat anti-Sca-1 (1:100) (BD Pharmingen ${ }^{\mathrm{TM}}$; San Jose, CA), Rabbit polyclonal anti-ALB (1:200) (DAKO), Rabbit anti-CK19 (1:200) (Abcam; Cambridge, MA), Rabbit anti-E-Cadherin (1:200) (Cell Signaling Technology) and Rabbit anti-Sox9 (1:400) (Abcam). Then the cells were stained with AlexaFlour conjugated secondary antibodies from Invitrogen. All images were recorded with a Nikon Microphot-FXA microscope equipped with an Optronics DEI 750 3-chip CCD camera or a Zeiss AxioCarvert fluorescence microscope system with an Axiocam digital camera. Images were acquired and processed using $\mathrm{Q}$ imaging and AxioVision software.

\subsection{In Vitro Differentiation of Sca-1+ HPCs}

To analyze the hepatic differentiation capacity of single HPCs, cells were plated at a density of 100 cells per $35 \mathrm{~mm}$ dish and cultured in 10\% FBS-supplemented medium overnight before being changed into chemically defined medium, CDM, which is defined as DMEM/F-12 supplemented with $2 \mathrm{mM}$ L-glutamine, $0.11 \mathrm{mM} 2-$ mercaptoethanol, $1 \mathrm{mM}$ non-essential amino acids, 0.5 $\mathrm{mg} / \mathrm{ml} \mathrm{BSA} \mathrm{(fraction} \mathrm{V)].} \mathrm{HPCs} \mathrm{were} \mathrm{cultured} \mathrm{in} \mathrm{CDM}$ with $20 \mathrm{ng} / \mathrm{mL}$ hepatocyte growth factor (HGF, R\&D Systems, Minneapolis, MN), $10 \mathrm{ng} / \mathrm{mL}$ oncostatin $\mathrm{M}$ (OSM, Sigma), $0.5 \mathrm{mg} / \mathrm{L}$ insulin-transferring-selenium (ITS, Sigma) and $1 \mu \mathrm{M}$ dexamethasone (Sigma). The medium was changed every 2 - 3 days. The total RNA was extracted after 10, 15 and 20 days of induction.

To induce cholangiocytic differentiation, $0.5 \mathrm{ml} \mathrm{ma-}$ trigel (Invitrogen) mixed with $0.5 \mathrm{ml}$ serum free DMEM/ F12 medium supplement was added into $35 \mathrm{~mm}$ dishes. After incubation in $37^{\circ} \mathrm{C}$ for $2 \mathrm{~h}, 5 \times 10^{3}$ cells were plated onto the matrigel and medium was replaced with fresh CDM supplement containing 2\% matrigel and 2 $\mathrm{mg} / \mathrm{ml}$ laminin with $10 \mathrm{ng} / \mathrm{ml}$ HGF every 3 days [14]. Total RNA was extracted after a 20 day incubation. First, confirm that you have the correct template for your paper size. This template has been tailored for output on the custom paper size $\left(21 \mathrm{~cm}^{*} 28.5 \mathrm{~cm}\right)$.

\subsection{Tumor Formation Assay and Cell Transplantation}

For tumor formation assays, up to $5 \times 10^{6}$ cells suspended in $100 \mu \mathrm{l}$ PBS were subcutaneously injected into the right and left lower flanks of 6 - 8 week old Rag2-/ $-\gamma$-/-mice $(n=10)$. HepG2 cells were injected as a control and mice were observed over 6 months.

To assess the differentiation capacity of Sca-1+ HPCs in vivo, $1 \times 10^{6}$ pMX-IRES-EGFP-transduced HPCs were injected into the spleens of C57BL/6 mice $(n=12$, 8 - 10 week old). Acute liver damage was induced by intraperitoneal injection of $250 \mathrm{mg} / \mathrm{kg}$ body weight acetaminophen (APAP, Sigma) in sterile PBS eight to twelve hours before transplantation. Mice were euthanized at four weeks or six weeks after cell implantation. Recipient livers were fixed in $4 \%$ phosphate-buffered paraformaldehyde overnight at $4{ }^{\circ} \mathrm{C}$ and embedded in paraffin. Frozen livers were cut to $10 \mu \mathrm{m}$ cryosections and observed under fluorescence microscope for EGFP expression. Paraffin-embedded liver sections in $5 \mu \mathrm{m}$ were stained with anti-GFP (EMD Millipore, Billerica, MA) and anti-ALB antibodies.

\section{RESULTS}

\subsection{Isolation and Characterization of HPCs from Normal Adult Mouse Liver}

Previously, we identified a Sca-1 positive, bipotent HPC population in the periportal region of normal mouse liver tissue. By two-step liver perfusion with collagenase and subsequent cell enrichment by MACS using Sca-1 antibody conjugated mini-magnetic beads, the Sca-1 positive HPC populations were purified [4]. The cells are routinely cultured with EGF and CHIR99021. The primary cultures consist of mainly epithelial-shaped cells mixed with fewer fibroblast-like cells. However, during a series of subpassage cultures, the Sca-1+ HPCs appear morphologically homogenous with epithelial shaped cells (Figures 1(A) and (B)). The 9th passage Sca-1+ HPCs were serially diluted and plated onto two 96-well tissue culture plates (one cell in $100 \mu \mathrm{l}$ culture medium per well) and 10 clones then selected and named numerically as: clone 9-1-HPC, 9-2-HPC, 9-3-HPC, ‥ 9-10-HPC. 
Cells were cultured in a humidified $5 \% \mathrm{CO}_{2}$ atmosphere at $37^{\circ} \mathrm{C}$ and the medium changed at regular time intervals. Two clones 9-1-HPC (Figures 1(C) and (D)), 9-8-HPC (Figures 1(E) and (F)), and mixture HPCs were characterized in the present studies. RT-PCR shows that the HPCs express Sca-1, liver related genes (including AFP, ALB, Transferrin, TAT, CK19, GGT, ApoAII, ApoCII and ApoE) and liver-enriched transcription factors (including GATA-4, HNF-1 $\alpha$, HNF-3 $\alpha$, HNF-6, HNF-4, $\mathrm{HNF}-1 \beta, \mathrm{HNF}-3 \beta$ and $\mathrm{C} / \mathrm{EBP}-\gamma)$ (Figure 1(G)).

To fully characterize the Sca-1+ HPCs and distinguish them from other cell fractions in the adult liver, cell surface profiles were analyzed using specific antibodies for hepatic progenitor cells (Sca-1, CD13 and Prominin-1), oval cells/biliary epithelial cells (CD24/EpCAM) and hematopoietic/endothelial cells (CD31, CD34, CD45, CD90 and CD117) by flow cytometry. The flow cytometric data of 9-8-HPCs at the 18th, 26th and 31st passages indicate that a majority of cells are continuously positive for Sca-1 $(61 \% \pm 0.12 \%)$; along with CD13 (12.7\% $\pm 0.02 \%)$, CD24 (22.8\% $\pm 0.95 \%)$, Prominin-1 $(16.4 \% \pm 0.19 \%)$ and EpCAM $(12.6 \% \pm$ $1.05 \%$ ) but do not express the endothelial cell and hematopoietic cell markers CD31, CD34, CD45, CD90 and CD117 (Figure 2). 9-1-HPC at the 16th, 22nd and 25th passages have similar expression patterns with 9-8 HPCs but are negative for EpCAM. Mixed HPCs at the 20th, 26th and 30th passages have similar expression pattern with 9-8 HPCs but with a lower percentage of cells ex- pressing Sca-1 $(34 \% \pm 0.06 \%)$ (Table 1).

We further examined the Sca-1+ HPCs using immunocytochemistry (ICC) and western blot analysis. ICC analysis shows that HPCs are positive for Sca-1, AFP, ALB and CK19 (Figure 3(A)). Western blot analysis confirms the expression of AFP, ALB and CK19 (Figure 3(B)). Meanwhile, we find that Sca-1+ HPCs also express E-Cadherin and Sox9 by ICC (Figures 3(C) and (D)) and RT-PCR (Figure 3(E)), markers which have been used as liver progenitor cell markers $[15,16]$. These results strongly suggest that Sca-1+ HPCs can be stably maintained during long-term in vitro culture.

\subsection{In Vitro Differentiation Potential of Sca-1+ HPCs}

To evaluate the potential of Sca-1+ HPCs to differentiate into hepatocyte-like cells, we used HGF, OSM, ITS and dexamethasone to promote maturation of HPCs. Sca-1+ HPCs were plated at ultra-low density(100 cells per $35 \mathrm{~mm}$ dish) and cultured with HGF for 5 days, followed by another 2 weeks of OSM, ITS and dexamethasone treatment in CDM. We analyzed colony-constituent cells after 10, 15 and 20 days of induction for expression of albumin and CK19. Sca-1+ HPCs coexpress albumin and CK19 before induction (Figure 4(A)). After 10 days of induction, half (50\%) of HPCs are only albumin-positive (Figure 4(B)). By 15 days of induction $90 \%$ of HPCs are albumin positive only, while $10 \%$ are
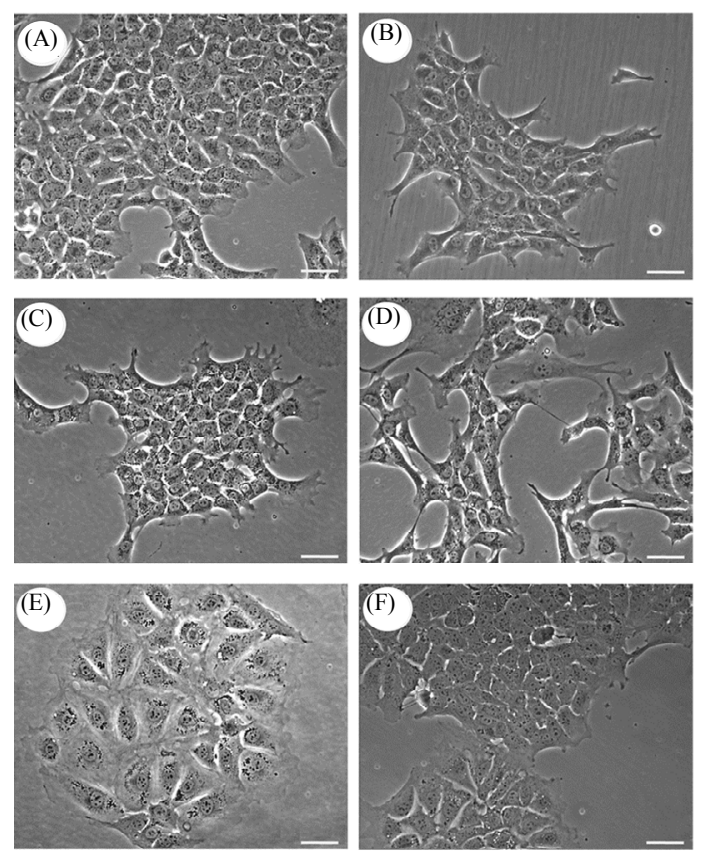

(G)

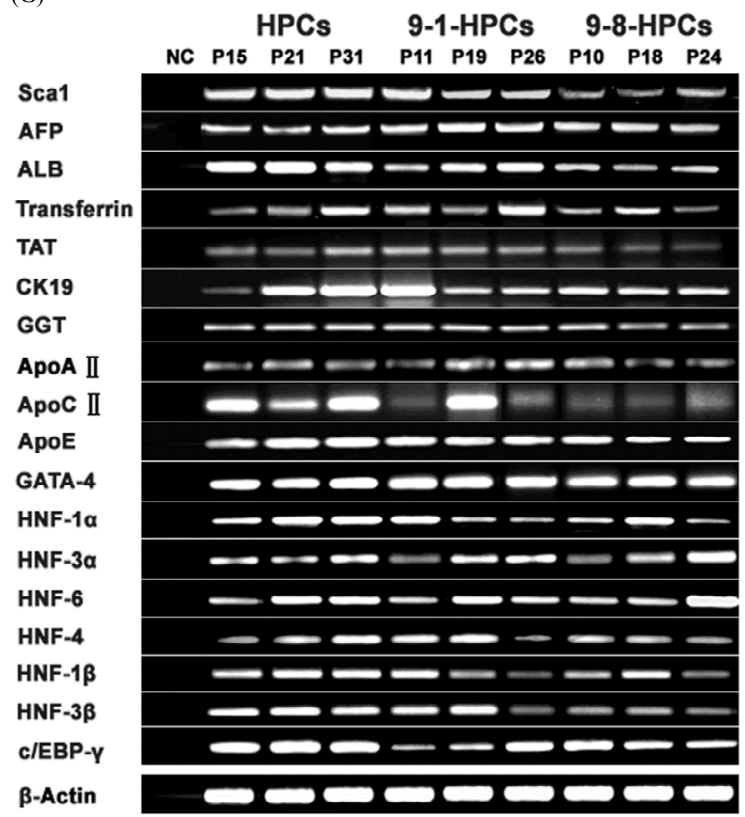

Figure 1. Establishment of HPC cell lines from wild type C57BL/6 mice. Phase contrast photographs of HPCs in passage 8 and 28, 9-1-HPCs in passage 7 and 18, 9-8-HPCs in passage 12 and 22 (Magnification, 200×) (A)-(F). Bars, $50 \mu \mathrm{m}$. RT-PCR analysis of selected transcription factors and liver-related genes in HPCs, 9-1-HPCs and 9-8-HPCs (G). NC, negative control. 

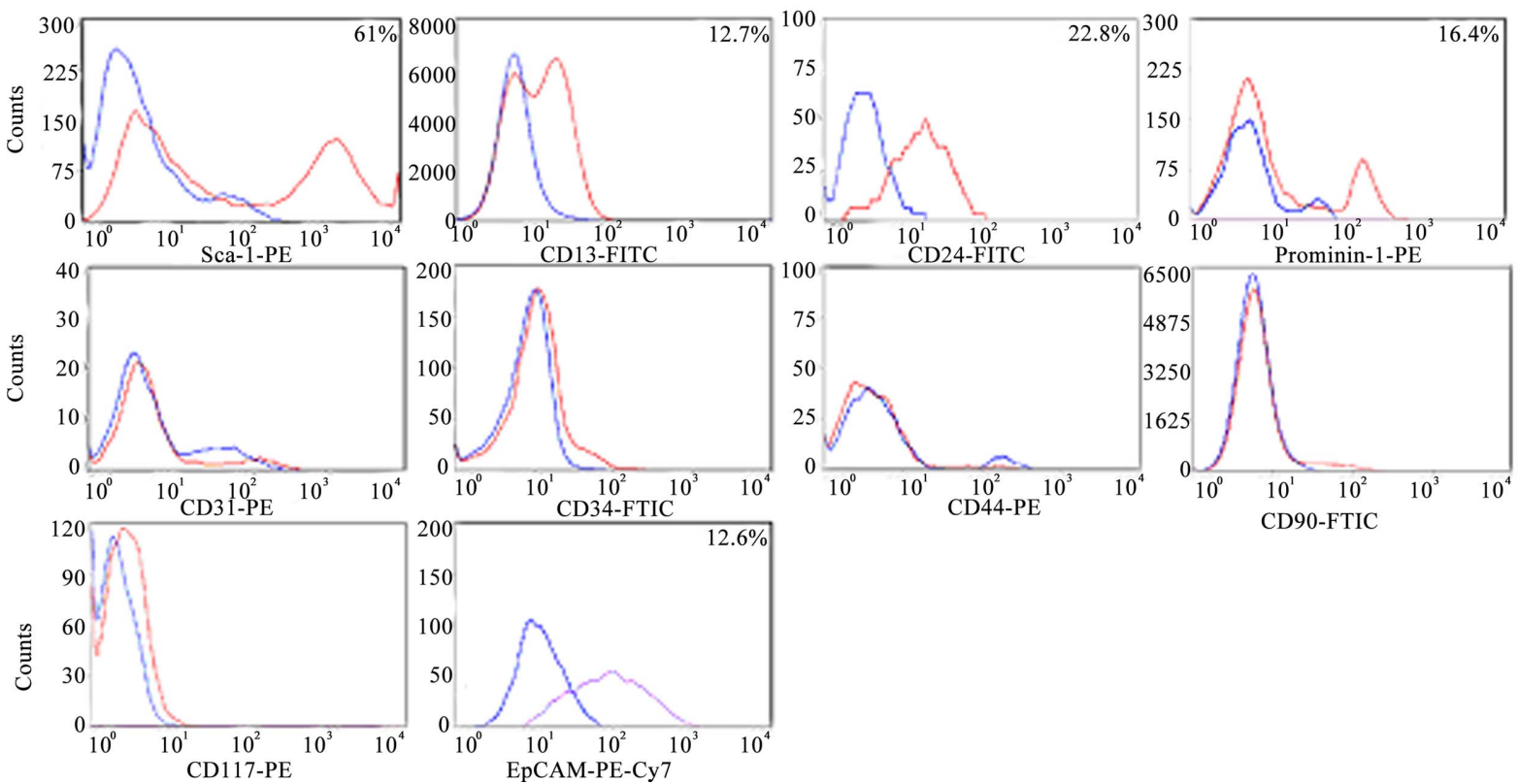

Figure 2. Identification of surface markers in HPCs by flow cytometry. Histograms represent the analyzed markers versus their corresponding isotype controls. Blue curve: fluorescence of isotype controls; Red/Purple curve: fluorescently labeled cells. The data shown represent the experiments using 9-8 HPCs.

(A)
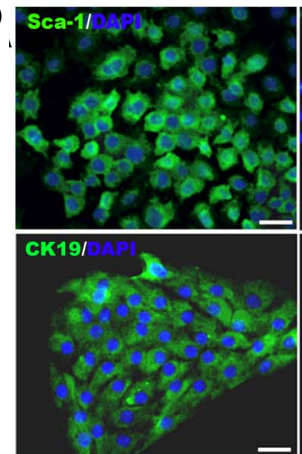

(B)

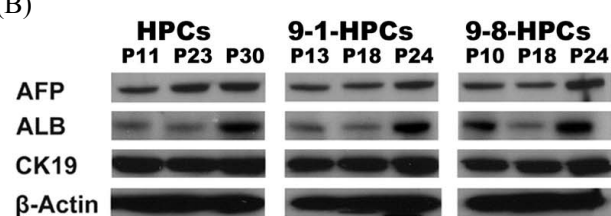

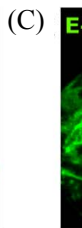

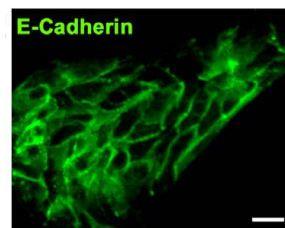

(D)

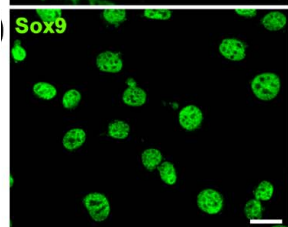

(E)

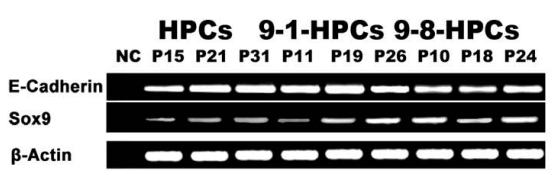

Figure 3. Phenotypic analysis of HPCs. Immunofluorescence analysis for Sca-1, ALB, CK19 and AFP (A). Protein was collected from different passage cells and analyzed for expression of AFP, ALB and CK19 (B). Immunofluorescence analysis of E-Cadherin and Sox9 (C) and (D). RT-PCR analysis of E-Cadherin and Sox9 (E). Data are from one representative of at least three independent experiments. Magnification: (A) 200×; (C) and (D) 400×. Bars: $50 \mu \mathrm{m}$. The nucleus was stained with DAPI.

only positive for CK19 (Figure 4(C)). Almost all HPCs are albumin-positive and CK19-negative after 20 days of induction (Figure 4(D)), which suggests that HPCs can differentiate into hepatocyte-like cells. Periodic acidSchiff staining of HPCs shows very weak staining before induction (Figure 4(E)) but become strongly positive after 20 days of induction (Figure 4(F)), indicating that HPCs can differentiate into mature hepatocytic cells with sufficient glycogen expression after 20 days of induction in culture. RNA analysis of the HPCs in hepatic induction medium for 20 days confirms up-regulation of hepatic lineage marker ALB, with a 10-fold increase after 20 days of induction. However, the expression of CK19 decreases slightly compared to initial control levels (Figures 4(G) and (H)).

When HPCs are cultured on Matrigel in CDM with 10 
Table 1. Expression of surface markers in HPCs, 9-1 HPCs and 9-8 HPCs.

\begin{tabular}{cccc}
\hline & HPCs & 9-1-HPCs & 9-8-HPCs \\
\hline Sca-1 & $34 \pm 0.06$ & $57 \pm 0.18$ & $61 \pm 0.12$ \\
CD13 & $29.6 \pm 0.17$ & $13.6 \pm 2.26$ & $12.7 \pm 0.02$ \\
CD24 & $27.8 \pm 0.75$ & $37.4 \pm 1.69$ & $22.8 \pm 0.95$ \\
Prominin-1 & $11.5 \pm 0.65$ & $11.4 \pm 0.4$ & $16.4 \pm 0.19$ \\
EpCAM & $19.4 \pm 0.25$ & negative & $12.6 \pm 1.05$ \\
CD31 & negative & negative & negative \\
CD34 & negative & negative & negative \\
CD45 & negative & negative & negative \\
CD90 & negative & negative & negative \\
CD117 & negative & negative & negative \\
\hline
\end{tabular}

ng/ml HGF, tubule-like branching morphogenesis appears after 10 days of plating (Figure 5(A)) and bile duct-like structures form 20 days latter (Figure 5(B)). While HPCs cultured on Matrigel in CDM without HGF do not form any branching or bile duct-like structures and most cells die after 20 days of plating (Figure 5(C)). Immunofluoresence analysis shows that bile duct-like structures are strongly positive for CK19 (Figure 5(D)) with a concomitant decrease in hepatocyte marker ALB expression after 20 days of plating (Figure 5(E)). RNA analysis of the HPCs in 3D Matrigel culture for 20 days reveals increased expression of biliary lineage marker CK19, aquaporin-1 (Aqp1), cystic fibrosis transmembrane conductance regulator (CFTR) and hairy and enhancer of split 1 (Hes1), and decreased expression of hepatocyte marker ALB (Figure 5(F)). These results indicate that HPCs have the capacity to differentiate into both hepatocyte-like cells and cholangiocyte-like cells.

Importantly, after transplantation of 20th passage HPCs into Rag2-/- $\gamma-/-$ mice, no tumors are detected in the mice after 6 months $(n=5)$ of continuous observation. Three control mice $(\mathrm{n}=5)$ transplanted with HepG2 cells formed tumors 3 weeks post injection, suggesting HPCs are non-tumorigenic.

\subsection{In Vivo Differentiation Potential of Sca-1+ HPCs}

To investigate the potential of HPCs to engraft and to differentiate within the microenvironment of the liver in vivo, we prepared recipient mice by using acetaminophen to injure the liver prior to cell transplantation [17]. HPCs labeled with EGFP were transplanted into APAPtreated mice. The mice were euthanized at 4 weeks $(n=6)$ or six weeks $(n=6)$ after implantation to detect transplanted cells. EGFP+ HPCs were detected in the recipient livers by fluorescent stereoscopic microscopy (Fig- ure 6(A)). Immunofluorescence analysis showed that HPCs differentiate into parenchymal heaptocytes that produce both EGFP and ALB (Figure 6(B)). The percentage of EGFP positive cell engraftment was $3.92 \% \pm$ $0.52 \%(n=6)$ at 4 weeks. There was no significant difference between 4 -week and 6-week engraftment. The donor-derived cells were observed mainly in liver parenchyma near the periportal area. The results confirmed that HPCs can differentiate into hepatocyte lineage in vivo after transplantation into recipient animals.

\section{DISCUSSION}

Hepatocyte transplantation has long been recognized as a potential treatment for life-threatening liver diseases. However, considering the severe shortage of usable primary hepatocytes and the limited proliferative nature of these valuable cells, a renewable stem cell source of

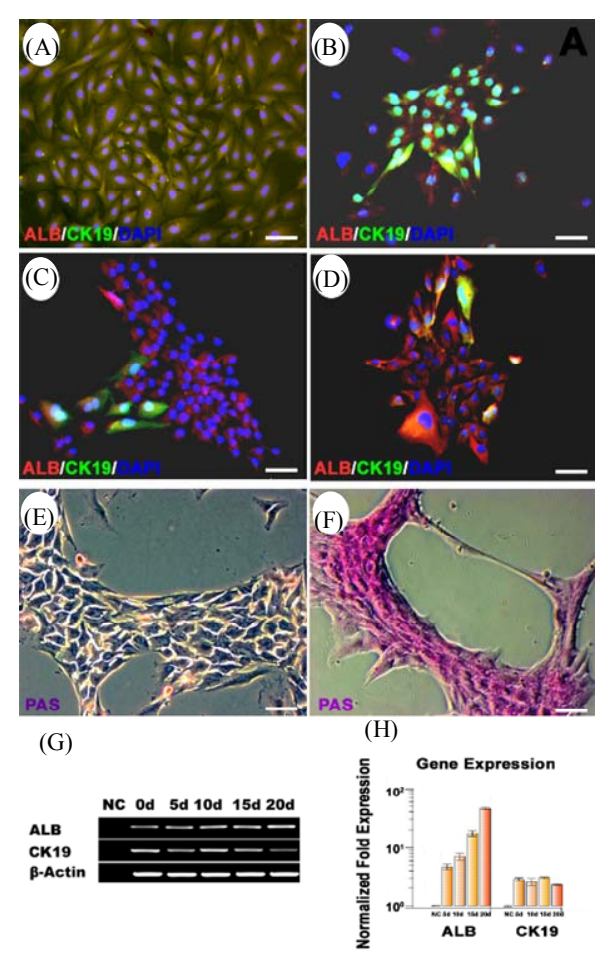

Figure 4. Hepatocyte differentiation of HPCs. Dual immunofluorescence stain of ALB and CK19 of HPCs before induction (A) and a single-cell source of HPCs cultured in chemical defined medium with $20 \mathrm{ng} / \mathrm{mL}$ HGF, 1 $\mu \mathrm{M}$ dexamethasone, $0.5 \mathrm{mg} / \mathrm{L}$ ITS and 10 $\mathrm{ng} / \mathrm{mL}$ OSM for 10 days (B), 15 days (C) and 20 days (D), the nucleus was stained with DAPI (Magnification, 200×). Periodic Acid Schiff (PAS) staining of HPCs before induction (E) and after 20 days induction (F). All bars, $50 \mu \mathrm{m}$. (G) and (H) RT-PCR and Realtime PCR analysis of HPCs that cultured in medium same as described above for 5, 10, 15 and 20 days. 

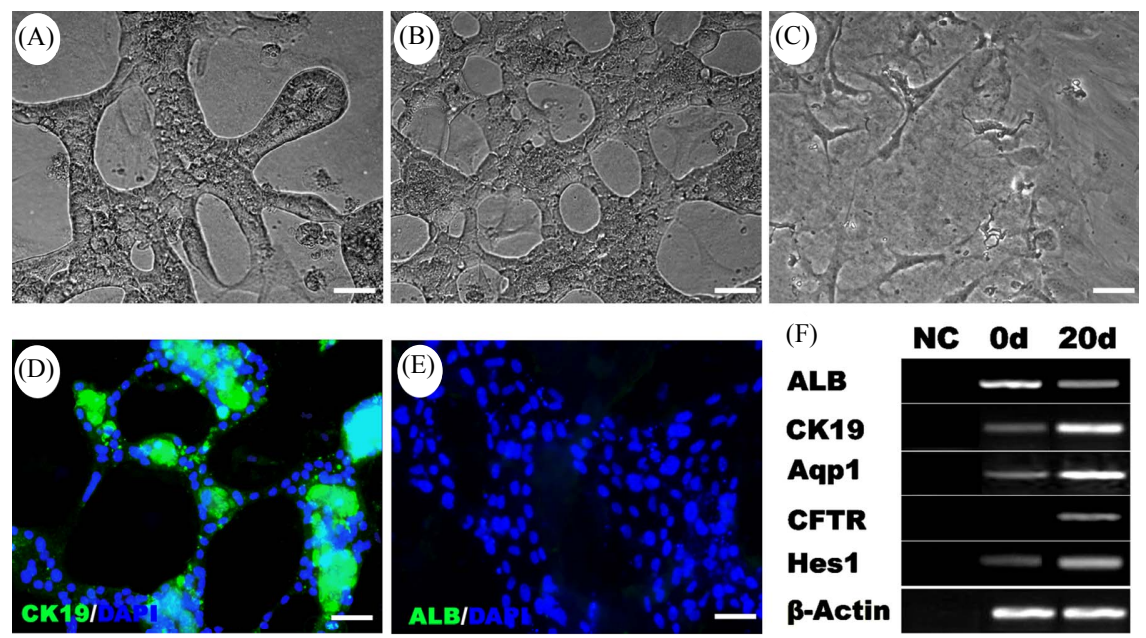

Figure 5. Differentiation of Sca-1+ HPCs into cholangiocyte-like cells. Sca-1+ HPCs showed branching morphogenesis (A) and bile duct-like structure (B) in 3-D matrigel culture after 10 and 20 days compared with control group (C). Immunofluorescence analysis for CK19 and ALB of HPCs cultured on 3D matrigel in CDM with $10 \mathrm{ng} / \mathrm{mL}$ HGF after 20 days, the nucleus was stained with DAPI (D)and (E). (F) RT-PCR analysis of cholangiocyte-related genes in HPCs after cultured on 3D matrigel for 20 days. Magnification, 200×; Bars, $50 \mu \mathrm{m}$.
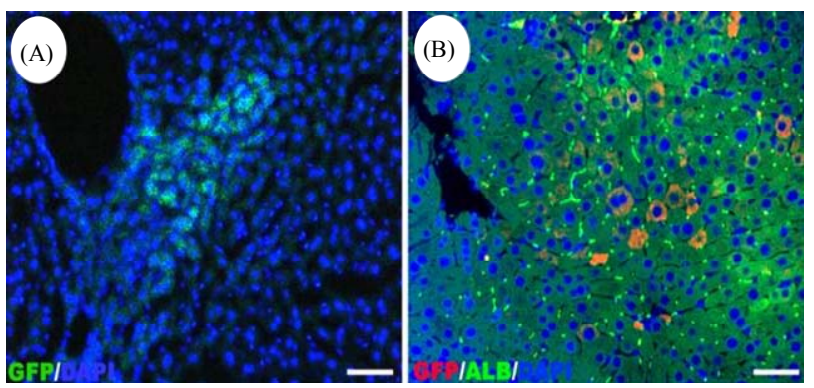

Figure 6. Differentiation of Sca-1+ HPCs in mice in vivo. (A) Fluorescent views of transplanted liver cryostat sections after 6 weeks of transplantation in vivo. (B) Immunofluorescence analysis of recipient liver sections. Transplanted Sca-1+ HPCs are positive for GFP (red) and ALB (green). The nucleus was stained with DAPI. Magnification, 200×; Bar, $50 \mu \mathrm{m}$.

hepatocytes is important for therapeutic transplant. Recent studies confirm that hepatic stem cells are resident in adult liver and play a critical role in mediating physiological and pathological liver regeneration [18]. However, despite extensive efforts, the isolation and maintenance of hepatic stem cells from liver has been hampered by the lack of specific surface markers and high efficiency culture systems.

Based on our previous studies with Sca-1, we further demonstrate in this study that Sca-1 can be utilized as a reliable surface marker to isolate liver progenitor cells from naïve murine adult liver tissues, and more importantly, these cells can be maintained in long-term culture using a combination of EGF and a small molecule GSK3 inhibitor CHIR99021. The proliferation of Sca-1+ HPCs is strictly dependent on the combination of EGF and
CHIR99021. Cell survival and growth are significantly retarded after the removal of CHIR99021 or EGF from the medium. Remarkably, even after long term expansion, cells are still competent to differentiate into mature hepatocytes or cholangiocytes efficiently in vitro and generate hepatocytes in vivo, suggesting the bipotential differentiation capability of the cells. Our study represents a novel paradigm to self-renew of hepatic progenitor cells. The cells can be used as a promising cell model to understand the molecular mechanisms involved in liver progenitor cell proliferation and differentiation. Moreover, it would be useful to test if the same regimen could also be used to expand human liver progenitor cells in the future.

Although the cells were purified using Sca-1 labeled MACS, we found the cultured HPCs do not homogenously express Sca-1 and other liver stem cell markers even after single cell cloning. This suggests that HPCs cultured under such conditions are a hierarchical cell population. Whether Sca-1 positive cells are progenitor cells that are responsible for long-term self-renewal of the cell population remains to be determined. Interestingly, we found HPCs under this culture condition also express Sox9, a gene labeling a liver progenitor pool within the peri-portal region. This population has been shown through in vivo lineage tracing to physiologically supply hepatocytes and bile duct cells [16]. This supports the notion that Sca-1 positive HPCs may be the in vitro counterpart of naïve liver progenitors. However, further studies are required to depict the lineage relationship of Sca-1 positive and Sox9 positive liver progenitors in situ. 


\section{ACKNOWLEDGEMENTS}

We thank Montserrat Caballero for providing cryostat, Carolyn Suitt for tissue section histology support, Barry Udis for flow cytometric analysis and the UNC Institutional Animal Care and Use Committee staff for help with animal work. We thank Microscopy Services Laboratory and Tissue Culture Facility for providing support.

\section{REFERENCES}

[1] Azuma, H., Hirose, T., Fujii, H., Oe, S., Yasuchika, K., Fujikawa, T. and Yamaoka, Y. (2003) Enrichment of hepatic progenitor cells from adult mouse liver. Hepatology, 37, 1385-1394. doi:10.1053/jhep.2003.50210

[2] Fougere-Deschatrette, C., Imaizumi-Scherrer, T., StrickMarchand, H., Morosan, S., Charneau, P., Kremsdorf, D., Faust, D.M. and Weiss, M.C. (2006) Plasticity of hepatic cell differentiation: Bipotential adult mouse liver clonal cell lines competent to differentiate in vitro and in vivo. Stem Cells, 24, 2098-2109. doi:10.1634/stemcells.2006-0009

[3] Wang, J., Clark, J.B., Rhee, G.S., Fair, J.H., Reid, L.M. and Gerber, D.A. (2003) Proliferation and hepatic differentiation of adult-derived progenitor cells. Cells Tissues Organs, 173, 193-203. doi:10.1159/000070375

[4] Wright, N., Samuelson, L., Walkup, M.H., Chandrasekaran, P. and Gerber, D.A. (2008) Enrichment of a bipotent hepatic progenitor cell from naive adult liver tissue. Biochemical and Biophysical Research Communications, 366, 367-372. doi:10.1016/j.bbrc.2007.11.129

[5] Lazaro, C.A., Croager, E.J., Mitchell, C., Campbell, J.S., Yu, C., Foraker, J., Rhim, J.A., Yeoh, G.C. and Fausto, N. (2003) Establishment, characterization, and long-term maintenance of cultures of human fetal hepatocytes. Hepatology, 38, 1095-106. doi:10.1053/jhep.2003.50448

[6] Kamiya, A., Kakinuma, S., Yamazaki, Y. and Nakauchi, H. (2009) Enrichment and clonal culture of progenitor cells during mouse postnatal liver development in mice. Gastroenterology, 137, 1114-1126, 1126 e1-e14.

[7] Dabeva, M.D., Alpini, G., Hurston, E. and Shafritz, D.A. (1993) Models for hepatic progenitor cell activation. Proceedings of the Society for Experimental Biology and Medicine, 204, 242-252. doi:10.3181/00379727-204-43660

[8] Wang, X., Foster, M., Al-Dhalimy, M., Lagasse, E., Finegold, M. and Grompe, M. (2003) The origin and liver repopulating capacity of murine oval cells. Proceedings of the National Academy of Sciences of USA, 100, 1188111888. doi:10.1073/pnas. 1734199100

[9] Theise, N.D., Saxena, R., Portmann, B.C., Thung, S.N., Yee, H., Chiriboga, L., Kumar, A. and Crawford, J.M. (1999) The canals of Hering and hepatic stem cells in hu- mans. Hepatology, 30, 1425-1433. doi: $10.1002 /$ hep. 510300614

[10] Jelnes, P., Santoni-Rugiu, E., Rasmussen, M., Friis, S.L., Nielsen, J.H., Tygstrup, N. and Bisgaard, H.C. (2007) Remarkable heterogeneity displayed by oval cells in rat and mouse models of stem cell-mediated liver regeneration. Hepatology, 45, 1462-1470. doi:10.1002/hep.21569

[11] Jin, C., Samuelson, L., Cui, C.B., Sun, Y. and Gerber, D.A. (2011) MAPK/ERK and Wnt/beta-Catenin pathways are synergistically involved in proliferation of Sca-1 positive hepatic progenitor cells. Biochemical and Biophysical Research Communications, 409, 803-807. doi:10.1016/j.bbrc.2011.05.094

[12] Ring, D.B., Johnson, K.W., Henriksen, E.J., Nuss, J.M., Goff, D., Kinnick, T.R., Ma, S.T., Reeder, J.W., Samuels, I., Slabiak, T., Wagman, A.S., Hammond, M.E. and Harrison, S.D. (2003) Selective glycogen synthase kinase 3 inhibitors potentiate insulin activation of glucose transport and utilization in vitro and in vivo. Diabetes, 52, 588595. doi: $10.2337 /$ diabetes.52.3.588

[13] Seglen, P.O. (1976) Preparation of isolated rat liver cells. Methods in Cell Biology, 13, 29-83. doi:10.1016/S0091-679X(08)61797-5

[14] O’Brien, L.E., Yu, W., Tang, K., Jou, T.S., Zegers, M.M. and Mostov, K.E. (2006) Morphological and biochemical analysis of Rac1 in three-dimensional epithelial cell cultures. Methods in Enzymology, 406, 676-691.

[15] Furuyama, K., Kawaguchi, Y., Akiyama, H., Horiguchi, M., Kodama, S., Kuhara, T., Hosokawa, S., Elbahrawy, A., Soeda, T., Koizumi, M., Masui, T., Kawaguchi, M., Takaori, K., Doi, R., Nishi, E., Kakinoki, R., Deng, J.M., Behringer, R.R., Nakamura, T. and Uemoto, S. (2011) Continuous cell supply from a Sox9-expressing progenitor zone in adult liver, exocrine pancreas and intestine. Nature Genetics, 43, 34-41. doi:10.1038/ng.722

[16] Nierhoff, D., Ogawa, A., Oertel, M., Chen, Y.Q. and Shafritz, D.A. (2005) Purification and characterization of mouse fetal liver epithelial cells with high in vivo repopulation capacity. Hepatology, 42, 130-139. doi:10.1002/hep.20735

[17] Quintana-Bustamante, O., Alvarez-Barrientos, A., Kofman, A.V., Fabregat, I., Bueren, J.A., Theise, N.D. and Segovia, J.C. (2006) Hematopoietic mobilization in mice increases the presence of bone marrow-derived hepatocytes via in vivo cell fusion. Hepatology, 43, 108-116. doi:10.1002/hep.21005

[18] Fukuda, A., Kawaguchi, Y., Furuyama, K., Kodama, S., Horiguchi, M., Kuhara, T., Koizumi, M., Boyer, D.F., Fujimoto, K., Doi, R., Kageyama, R., Wright, C.V. and Chiba, T. (2006) Ectopic pancreas formation in Hes1knockout mice reveals plasticity of endodermal progenitors of the gut, bile duct, and pancreas. Journal of Clinical Investigation, 116, 1484-1493. doi:10.1172/JCI27704 